\title{
A STATE OF ART FOR TRAFFIC REROUTING BASED ON POLLUTION INTENSITY
}

Chahat Agarwal ${ }^{1}$, Akshay $^{2}$, Priya Vijay ${ }^{3}$

Abstract- Nowadays, Air Pollution and population health is one of the most important environmental and public health issues. Economic development, urbanization, energy consumption, transportation/motorization and rapid population growth are major driving forces of air pollution in large cities (especially in megacities). Since people in the current world are more dependent on handheld systems and to enable the development of Smart City we need IoT enabled device which would help in order to navigate the routes based on pollution intensity. The paper briefly studies the existing ways as a survey to identify the pollution and to control the traffic controlling and rerouting mechanism to control pollution aggravating through traffic in congested cities

Keywords - Survey, Intimation, Pollution Intensity, Route Optimization, IOT

\section{INTRODUCTION}

Smart City is an urban related development scheme. The main vision of the smart city is to integrate the Information and Communication Technology with Internet of Things (IoT). Integration of Internet of Things (IoT) is an important factor here since; if we are aiming on developing a smart city then it should be ensured that most of the assets have to be connected to the cloud through the means of Internet. With the help of this various set of data can be monitored and analyzed simultaneously and Output can be generated in no time.

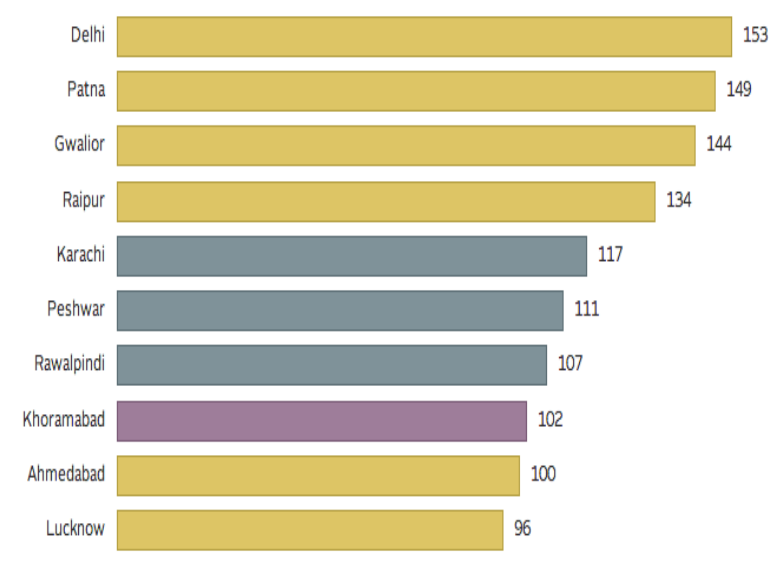

Source: World Health Organization

Figure 1. Present pollution rate

The Figure 1 is taken from Google Images which tells about the pollution rate statistics from WHO. It shows the pollution rate for each city in India because of traffic in the cities.

The above graph shows the necessity of pollution control mechanism and dwells a motivation to investigate the various means of techniques to control traffic pollution in our major cities. A smart city must be able to adapt the latest technologies in order for it to be smart. The poor quality of air has increased the respiratory diseases like asthma and bronchitis which in turn increased the risk of lot of diseases like cancer, and creates lot of problems to our health care system. The paper describes and analyses the various existing technologies to traffic rerouting by absorbing air pollution through IOT and a proposed architecture with intelligence for optimization in rerouting of traffic in congested cities of India.

${ }^{1}$ UG Student, Dep. of Computer Science and Engineering, Rajalakshmi Engineering College, Chennai, Tamil Nadu, India

${ }^{2}$ UG Student, Dep. of Computer Science and Engineering, Rajalakshmi Engineering College, Chennai, Tamil Nadu, India

${ }^{3}$ Associate Prof., Dep. of Computer Science and Engineering, Rajalakshmi Engineering College, Chennai, Tamil Nadu, India 


\section{STATE OF ART}

2.1 Air Pollution Detection and Control Control in Vehicles -

This system implements a concept of switching of the vehicle when the pollution goes beyond a Critical level. Car is running on the factor of burning fuels so it's not appropriate to switch off the vehicle when it creates some pollutant gases. [1]

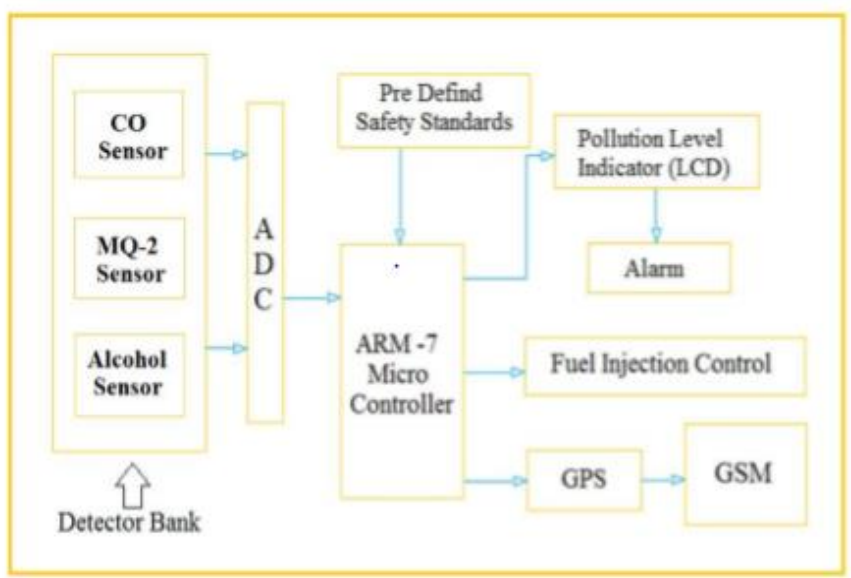

Figure 2. Block diagram of system

The Figure 2 is referred from the paper, [1]"Automated System for Air Pollution Detection and Control in Vehicles" by Anita Kulkarni, P. Ravi Teja

The sensors cannot be deployed in the vehicles as it may produce very high value in PPM.

Only by using a stationary gas sensor to sense the pollution rate of a particular area we can identify the pollution rate in that particular area.

\subsection{Smart Pollution Detection and Tracking System Embedded with AWS IoT Cloud -}

In this system the pollution of different places is identified using a vehicle for every $20 \mathrm{~km}$. But the problem is that there are no vehicles which are not mobile and for effective pollution identification we need a fixed locality monitoring system [2].

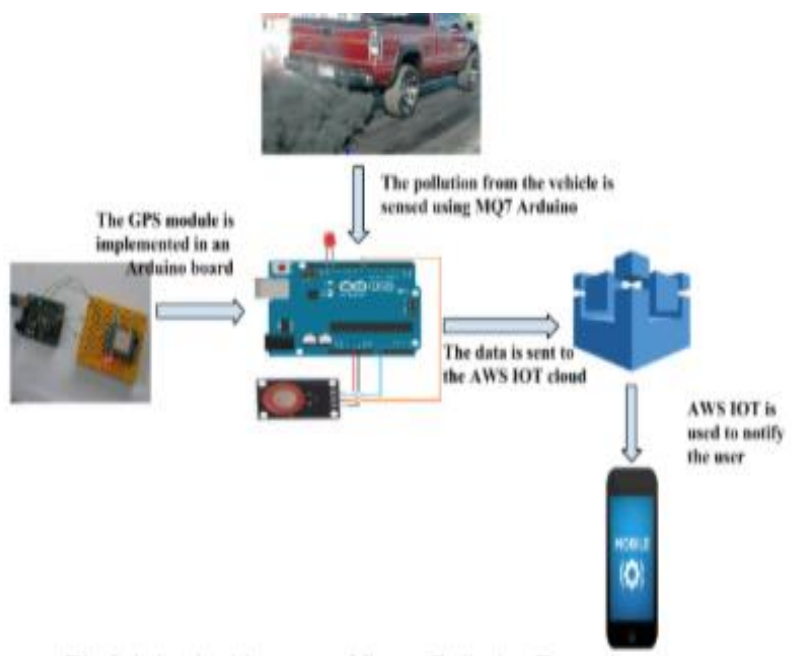

Figure 3. Block diagram of the system

The Figure 3 is referred from the paper, [2] "Smart Pollution Detection and Tracking System Embedded with AWS IoT Cloud" by Marina Shruthi M, Dr. L. Josephine Mary.

We need to have a stationary sensor to sense the values of that particular place, using a pi board to integrate with the gas sensor where we can sense the data in the atmosphere and implement in the cloud for easy access of data

\subsection{Participatory Air Pollution Monitoring using Smartphones-}

In this project the detection modules are attached with a smartphone physically and the monitoring is done. But in order to incorporate more sensors or modules a single mobile is not sufficient. We need a mini microcontroller for this purpose [3]. 


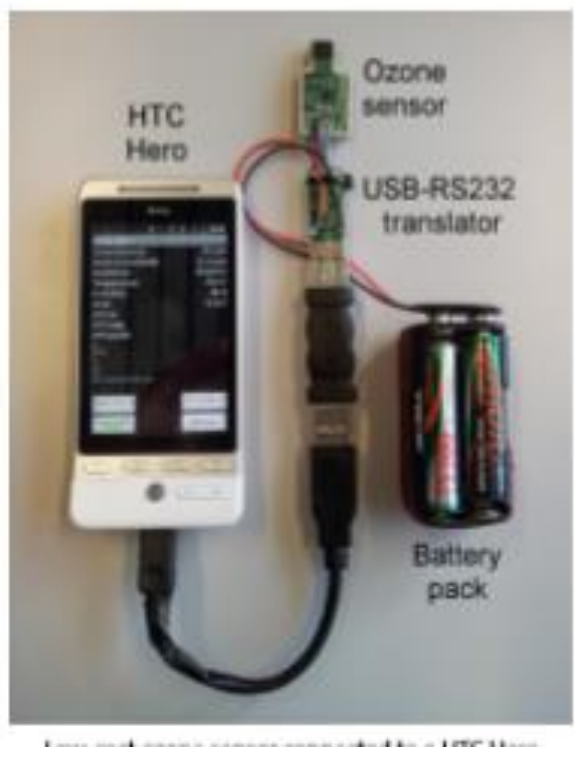

Figure 4. Block diagram of the system

The Figure 4 is referred from the paper, [3] "Participatory Air Pollution Monitoring uses Smartphones" by David Hasenfratz, Olga Saukh Silvan Sturzenegger and Lothar Thiele.

We have used a pi board connected to a gas sensor to obtain the readings which is sent to the database, we can use a smartphone application to access the pollution rate of different areas.

\section{PROPOSED ARCHITECTURE}

The sensor devices are placed in several areas. Mainly it is placed in certain areas where the pollution is said to be higher than the normal level. These devices sense the pollution level of the particular area continuously and it sends these accumulated values to the different access points using Wireless Sensor Networks (WSN).

The access points which holds the data of different areas pushes these data to the database. Now the database contains the pollution levels of different places. Here we use Amazon Web Service (AWS) as the database.

This is the basic Architecture Diagram,

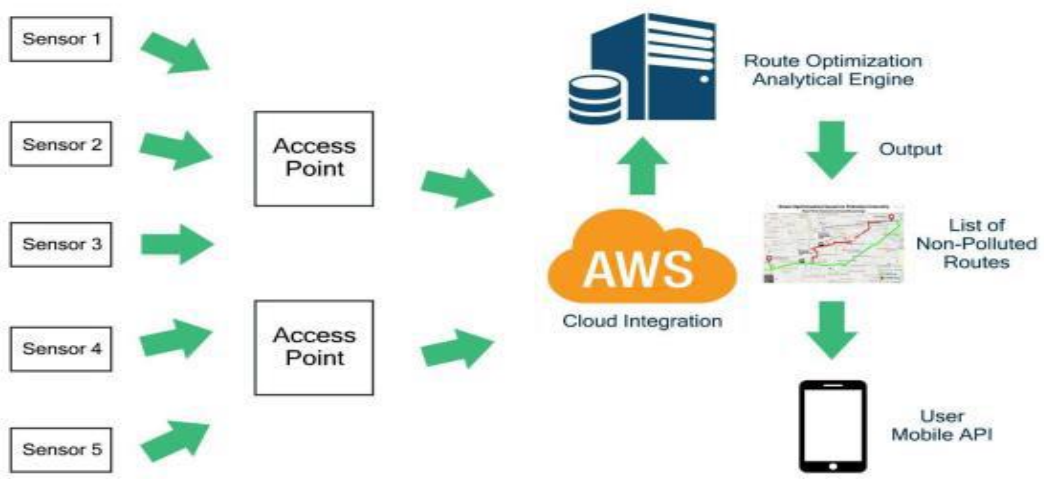

Figure 5. Proposed Architecture

The Figure 5 represents the basic Architecture Diagram for our Project. The sensor data will be collected from various places in the heavy traffic route cities. It will be analyzed and the traffic will be rerouted in the ways which are free and minimal pollution rate so that traffic pollution rate can be avoided in increasing in a particular area.These data are now transferred to the Route Optimization Analytical Engine (ROAE) to find a optimized route for travelers that should not increase the journey time for the travelers to reach their destination.Here all the analytical processing is performed with the obtained data and using this optimized route is identified.

These can be accessed using a smartphone application, when the users use the application in smartphone to view the pollution free route which is obtained from the Route Optimization Analytical Engine. By using a simple smartphone application we can create an alert for people 


\section{CONCLUSION}

The proposed project focuses on intimating the people about the environment in which they sustain. Even though pollution control measures are introduced by the government it is always better to have a self-prevention policy. The monitoring system monitors about the environment conditions of the place and if the pollution level is higher, then it alerts the user. Alertness given to the user helps them to make necessary arrangements to prevent themselves. It is always said "Prevention is better than cure". Hence the proposed project gives an efficient solution for the prevention of people from pollution.

\section{REFERENCES}

[1] Anita Kulkarni, P. Ravi Teja. Automated System for Air Pollution Detection and Control in Vehicles. International Journal of Advanced Research in Electrical, Electronics and Instrumental Engineering. Volume 3, Issue 9, September 2014.

[2] Marina Shruthi M, Dr. L. Josephine Mary. Smart Pollution Detection and Tracking System Embedded with AWS IoT Cloud. International Journal of Advanced Research in Computer Science and Software Engineering. Volume 6, Issue 4, April 2016.

[3] David Hasenfratz, Olga Saukh Silvan Sturzenegger and Lothar Thiele. Participatory Air Pollution Monitoring using Smartphones.

[4] P. Ribeiro, J.F Mendes. The Influence of Air Pollution in the Selection of Healthy Routes for Walking and Cycling.

[5] Robert D. Allison, III. Air pollution control system and method. Google patents, 31 May 2016.

[6] Aaron Joseph Teller. Process for removing particulates from a gas. Google patens, 18 May 1976.

[7] Kilpatrick Myron O. Recovery of carbon black from suspension in a gas. Google patens, 12 Feb 1952 .

[8] Illingworth Robert H. Air purification. Google patens, 20 Jun 1967.

[9] David E. Sterling. Wind tunnel for erosion testing. Google Patens, 14 Nov 2017.

[10] James P. Cox, Kelly K. Cox. Gas scrubbing apparatus and process. Google patens, 29 Mar 1988.

[11] H. Forbes Davis. Modular air scrubber system. Google Patens, 14 Aug 1990.

[12] Raymond T. Fong. Process for removing volatile organic materials. Google patens, 16 Feb 1993.

[13] Niels Jacobsen, Henrik Maimann, Bjarne Rasmussen, Christian Holm Fridberg, Spray dryer absorption process for flue gas with entrained coarse particles, Google Patens, 16 Jun 2011.

[14] Kent Lückstädt Nielsen, An air disperser for spray-drying, and a method for manufacturing an air disperser comprising metal forming, Google Patens, 6 Apr 2017.

[15] Kljueva G, Lamm E, Shteinfeld B, Device for introducing a gaseous agent, Google Paten, 16 Apr 1974. 